\title{
Measurement of top quark properties in single top production
}

\section{Andres Tiko* on behalf of the CMS collaboration}

National Institute of Chemical Physics and Biophysics

E-mail: andres.tiko@cern.ch

\begin{abstract}
Single top topologies are exploited for studies of top quark properties. This includes the first measurement of single top polarization in the t-channel production mode in pp collisions, which directly confirms the $\mathrm{V}$-A nature of the $\mathrm{tWb}$ production vertex. W-helicity fractions are measured in the phase space sampled by a selection optimized for t-channel single top production, orthogonal to the $t \bar{t}$ final states used in traditional measurements of these properties. Anomalous tu(c) $\gamma$ and $\mathrm{tu}(\mathrm{c}) \mathrm{g}$ couplings of the top quark are searched for in t-channel single top production with multivariate analyses.
\end{abstract}

The European Physical Society Conference on High Energy Physics 22-29 July 2015

Vienna, Austria

\footnotetext{
* Speaker.
} 


\section{Introduction}

Top quark decays before hadronization and its spin information is measurable through angular distributions of its decay products. The study of the single top quark production provides a possibility to investigate many aspects of top quark physics that cannot be easily studied in t⿱亠幺 production, for example studying the Wtb vertex in a search for new interactions, flavour changing neutral current (FCNC) couplings in the production and decay processes, and a direct measurement of the CKM matrix element $V_{t b}$, providing a test of the standard model (SM). At the LHC, t-channel is the dominant single-top production mechanism with $\approx 80 \%$ of the cross section, the rest is provided by the $s$-channel and $\mathrm{W}$-associated production. All analyses presented here use $t$-channel as the signal process and are performed with the CMS detector [1].

The $t$-channel signature is shown on Figure 1. We are looking at leptonic decays of the $\mathrm{W}$ boson, which means we have one lepton in the final state along with considerable missing energy from the neutrino. There is also a b-jet from the top decay and a light spectator jet. There is a second b-jet which is usually outside our acceptance.

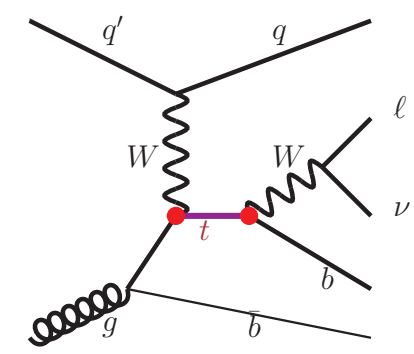

Figure 1: Single top $t$-channel production and leptonic decay.

\section{W boson helicity measurement}

As the first analysis to be discussed, we have measured the $\mathrm{W}$ boson helicity in single top production [2]. The polarization of $\mathrm{W}$ bosons from top quark decays is sensitive to non-SM Wtb couplings. W bosons can be produced with left-handed, longitudinal, or right-handed helicity. $\Gamma(\mathrm{t} \rightarrow \mathrm{Wb})=\Gamma_{\mathrm{L}}+\Gamma_{0}+\Gamma_{\mathrm{R}}$ holds for the corresponding partial widths. Helicity fractions are defined as $F_{i}=\Gamma_{i} / \Gamma$, where $i=L, 0$, or $R$, with $\sum F_{i}=1$. Experimental results extracted using $t \bar{t}$ events are in good agreement with SM predictions, this is the first measurement with single top events.

The analysis strategy is to measure the helicity fractions using the helicity angle $\cos \theta_{\ell}^{*}$, which is defined as the angle between the $\mathrm{W}$ boson momentum in the top quark rest frame and the momentum of the down-type decay fermion in the rest frame of the $\mathrm{W}$ boson. In addition to single top $t$-channel, our selection also contains a significant contribution from $t \bar{t}$ events. As the physics information contained in them is them is the same, and the selection is complementary to the CMS $\mathrm{t} \bar{t}$ analyses, we use these events as part of the signal.

The probability distribution of the helicity angle is shown in Eq. (2.1). According to the equation, we can obtain the helicity fractions by fitting to the measured distribution, which is 
shown on Figure 2.

$$
\rho\left(\cos \theta_{\ell}^{*}\right) \equiv \frac{1}{\Gamma} \frac{d \Gamma}{d \cos \theta_{\ell}^{*}}=\frac{3}{9}\left(1-\cos \theta_{\ell}^{*}\right)^{2} F_{L}+\frac{3}{4} \sin ^{2} \theta_{\ell}^{*} F_{0}+\frac{3}{8}\left(1+\cos \theta_{\ell}^{*}\right)^{2} F_{R}
$$
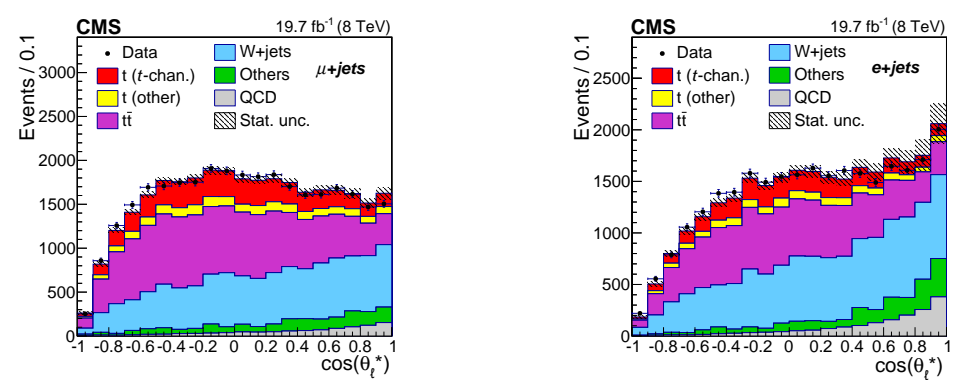

Figure 2: Measured distribution of the helicity angle. Muon channel on the left, electrons on the right.

The measurement is performed for the muon and electron channels separately as well as by combining the two channels. For the combined case we get $\mathrm{F}_{\mathrm{L}}=0.298 \pm 0.028$ (stat) \pm 0.032 (syst), $\mathrm{F}_{0}=0.720 \pm 0.039$ (stat) \pm 0.037 (syst), and $\mathrm{F}_{\mathrm{R}}=-0.018 \pm 0.019$ (stat) \pm 0.011 (syst), which is compatible with SM prediction. The negative value for $F_{R}$ comes from the requirement that the helicity fractions sum to 1 . The results for the muon and electron channel separately are compatible with the combined result. The results are illustrated on Figure 3.
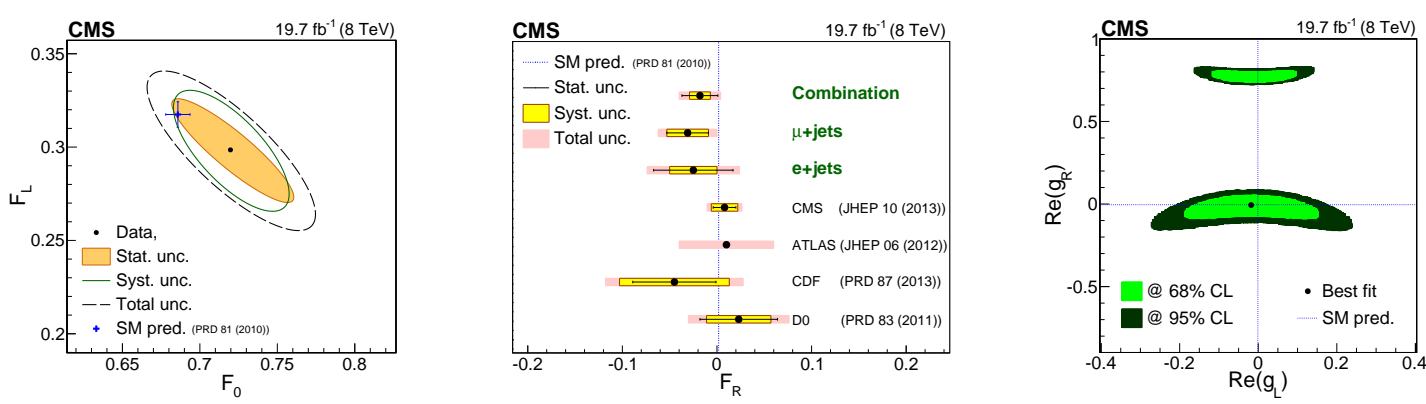

Figure 3: On the left, the results in $\mathrm{F}_{0}-\mathrm{F}_{\mathrm{L}}$ plane. In the middle, the value of the right-handed helicity fraction is compared to previous measurements. On the right, limits for anomalous gluon tensor couplings in the Wtb vertex.

We also set limits on the anomalous gluon couplings in the Wtb vertex according to the Lagrangian:

$$
\mathscr{L}_{t W b}^{\text {anom. }}=-\frac{g}{\sqrt{2}} \bar{b} \gamma^{\mu}\left(V_{L} P_{L}+V_{R} P_{R}\right) t W_{\mu}^{-}-\frac{g}{\sqrt{2}} \bar{b} \frac{i \sigma^{\mu v} q_{v}}{m_{W}}\left(g_{L} P_{L}+g_{R} P_{R}\right) t W_{\mu}^{-}+\text {h.c. }
$$

This is done by using helicity fractions as input for the TOPFIT program [3]. We assume SM values for the vector couplings $\left(\mathrm{V}_{\mathrm{L}}=1\right.$ and $\left.\mathrm{V}_{\mathrm{R}}=0\right)$ and set limits on the tensor couplings. The results are shown on the right panel in Figure 3. The best fit values are -0.017 for $\mathrm{g}_{\mathrm{L}}$ and -0.008 for $g_{R}$, which are in agreement with the SM prediction. 


\section{Top quark polarization measurement}

In single top quark production via $t$-channel, SM predicts that top quarks are produced $\approx 100 \%$ polarized through the V-A coupling structure along the momentum of the spectator quark that recoils against the single top quark. New physics models may lead to a depolarization in production or decay by altering the coupling structure.

The value we measure in the analysis [4] is the top quark spin asymmetry, which is related to polarization as shown in Eq. (3.1).

$$
A_{\ell}=\frac{1}{2} \cdot P_{t} \cdot \alpha_{\ell}=\frac{N(\uparrow)-N(\downarrow)}{N(\uparrow)+N(\downarrow)}
$$

Here $P_{t}$ is single top quark polarization along the chosen axis, $\alpha_{X}$ is the spin-analyzing power of a decay product $X$, i.e. the degree of correlation of its angular distributions with respect to the spin of the top quark. The value of the spin-analyzing power equals 1 in the SM for charged leptons, which we use in the measurement, but is in general modified by anomalous top quark couplings that can arise through an effective extension of the coupling structure at the Wtb vertex. $N(\uparrow)$ and $N(\downarrow)$ are the number of charged leptons aligned or counter-aligned with the direction of the spectator quark recoiling against the single top quark in the top-quark rest frame.

We select a high-purity sample of $t$-channel single top events and estimate the signal and background components by a fit to data. For fitting we use a boosted decision tree (BDT) discriminant, which is also used to reject background events. After cutting on the BDT, we use an angular observable sensitive to polarization and apply an unfolding techinque to infer the parton-level distribution. The angular distribution we use is of $\cos \theta_{X}^{*}$, which is defined as the angle between the lepton and the light jet in the reconstructed top quark rest frame.

The general form of the angular distribution $\theta_{X}^{*}$ of decay product $X=(W, \ell, v, b)$ in the top quark rest frame can be expressed as Eq. (3.2).

$$
\frac{1}{\sigma} \frac{\mathrm{d} \sigma}{\mathrm{d} \cos \theta_{X}^{*}}=\frac{1}{2}\left(1+P_{t} \alpha_{X} \cos \theta_{X}^{*}\right)=\left(\frac{1}{2}+A_{X} \cos \theta_{X}^{*}\right)
$$

At parton-level we expect a slope which corresponds to the asymmetry. The reconstructed distributions are shown on Figure 4.
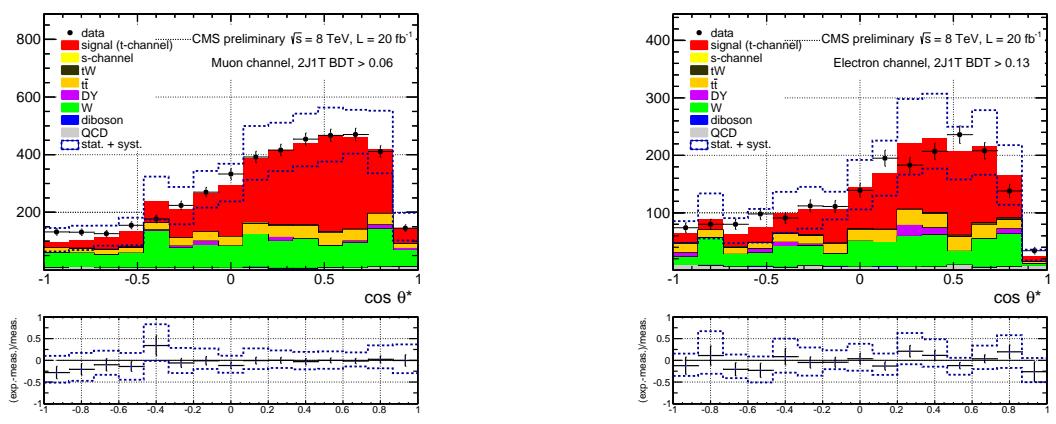

Figure 4: The reconstructed distributions of $\cos \theta^{*}$, on the left for muons, on the right for electrons. 
The unfolded distributions for muons and electrons are shown in Fig 5. From the unfolded distribution we extract the spin asymmetry of the top quark according to Eq. (3.1). They are combined with the BLUE method [5], giving an asymmetry of $A_{\ell}=0.41 \pm 0.06$ (stat.) \pm 0.16 (syst.), which corresponds to $P_{t}=0.82 \pm 0.12$ (stat.) \pm 0.32 (syst.) under assumption $\alpha_{\ell}=100 \%$. The result is compatible with SM. The main contribution to the result comes from the muon channel because electron channel has larger uncertainties.
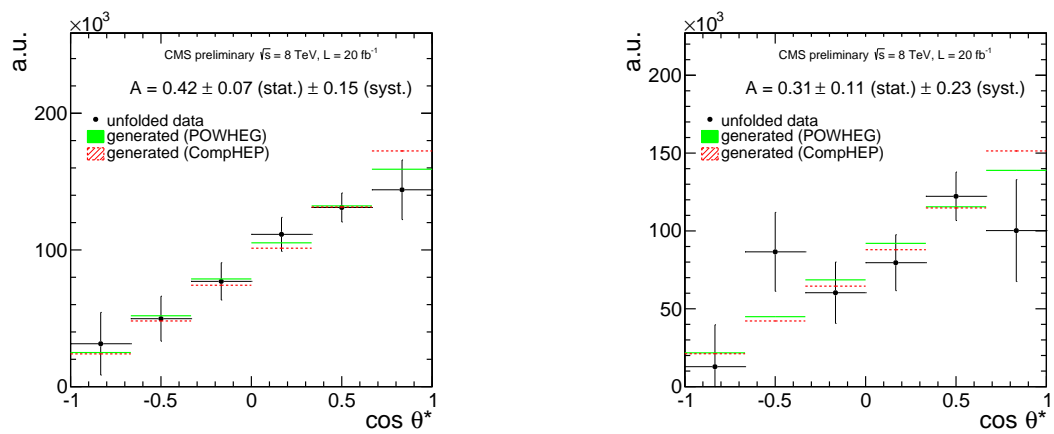

Figure 5: Unfolded distributions for muons on the left and electrons on the right.

\section{Anomalous Wtb couplings and FCNC measurements}

\subsection{Single top quark production in association with a photon}

The most general effective Lagrangian up to dimension-six in the vertex of $\operatorname{tu}(\mathrm{c}) \gamma$ is shown as Eq. (4.1).

$$
\mathscr{L}_{\text {eff }}=-e Q_{\mathrm{t}} \sum_{\mathrm{q}=\mathrm{u}, \mathrm{c}} \overline{\mathrm{q}} \frac{i \sigma^{\mu v} q_{v}}{\Lambda}\left(\kappa_{\mathrm{tq} \gamma}^{\mathrm{L}} P_{\mathrm{L}}+\kappa_{\mathrm{tq} \gamma}^{\mathrm{R}} P_{\mathrm{R}}\right) \mathrm{t} A_{\mu}+\text { h.c. },
$$

where $\kappa_{\mathrm{tq} \gamma}^{\mathrm{L}, \mathrm{R}}$ are the strengths of anomalous couplings. For simplicity, in analysis [6] we assume $\kappa_{\mathrm{tq} \gamma}^{\mathrm{L}}=\kappa_{\mathrm{tq} \gamma}^{\mathrm{R}}=\kappa_{\mathrm{tq} \gamma}$. In SM, the values of $\kappa_{\mathrm{tu} \gamma}$ and $\kappa_{\mathrm{tc} \gamma}$ are zero at tree level for both $\mathrm{u}$ and $\mathrm{c}$ quarks.

The final state we measure contains a single top quark and a photon, which has high $p_{T}$ because of the recoil against the heavy top quark. We consider only top quark decays in the muon channel. The decay chain is represented on Figure 6.

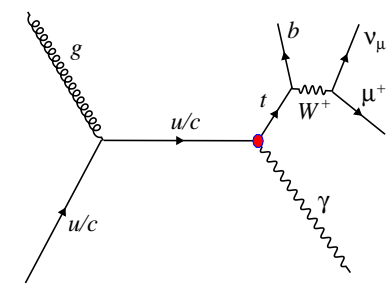

Figure 6: $\operatorname{tu}(\mathrm{c}) \gamma$ event topology.

Contributions of $W \gamma+$ jets and $W+$ jets backgrounds are estimated from data using a template fit method, others from simulation. Multivariate classification is used to optimize the discrimination 


\begin{tabular}{|c|c|c|c|c|}
\hline & Exp. limit (LO) & Obs. limit (LO) & Exp. limit (NLO) & Obs. limit (NLO) \\
\hline$\sigma_{t u \gamma} \times B r\left(W \rightarrow l v_{l}\right)$ & $0.0404 \mathrm{pb}$ & $0.0234 \mathrm{pb}$ & $0.0408 \mathrm{pb}$ & $0.0217 \mathrm{pb}$ \\
$\sigma_{t c \gamma} \times B r\left(W \rightarrow l v_{l}\right)$ & $0.0411 \mathrm{pb}$ & $0.0281 \mathrm{pb}$ & $0.0410 \mathrm{pb}$ & $0.0279 \mathrm{pb}$ \\
$\kappa_{t u \gamma}$ & 0.0367 & 0.0279 & 0.0315 & 0.0229 \\
$\kappa_{t c \gamma}$ & 0.113 & 0.094 & 0.0790 & 0.0652 \\
$B r(t \rightarrow u \gamma)$ & $0.0279 \%$ & $0.0161 \%$ & $0.0205 \%$ & $0.0108 \%$ \\
$B r(t \rightarrow c \gamma)$ & $0.261 \%$ & $0.182 \%$ & $0.193 \%$ & $0.132 \%$ \\
\hline
\end{tabular}

Table 1: Limits for tq $\gamma$ couplings

between SM and possible signal events, and trained for tu $\gamma$ and tc $\gamma$ separately. The distributions are shown on Figure 7.
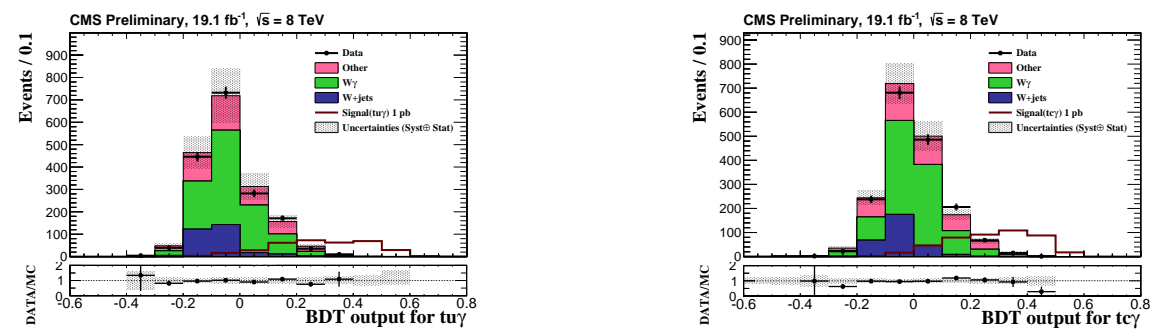

Figure 7: Signal distributions (scaled to a cross section of $1 \mathrm{pb}$ including the top quark leptonic decay branching ratio) compared to backgrounds. tu $\gamma$ on the left, tc $\gamma$ on the right.

The limits we obtain for the couplings are shown in Table 1. The experimental upper limits are getting close to the predictions of new physics models.

\subsection{Anomalous Wtb and FCNC tu(c)g couplings}

The most general, lowest dimension, CP conserving Lagrangian for the Wtb vertex is shown in $\mathrm{Eq}(4.2)$.

$$
\mathfrak{L}=-\frac{g}{\sqrt{2}} \bar{b} \gamma^{\mu}\left(f_{\mathrm{V}}^{\mathrm{L}} P_{\mathrm{L}}+f_{\mathrm{V}}^{\mathrm{R}} P_{\mathrm{R}}\right) t W_{\mu}^{-}-\frac{g}{\sqrt{2}} \bar{b} \frac{i \sigma^{\mu v} \partial_{v} W_{\mu}^{-}}{M_{\mathrm{W}}}\left(f_{\mathrm{T}}^{\mathrm{L}} P_{\mathrm{L}}+f_{\mathrm{T}}^{\mathrm{R}} P_{\mathrm{R}}\right) t+h \cdot c
$$

Here $f_{\mathrm{V}}^{\mathrm{L}}\left(f_{\mathrm{V}}^{\mathrm{R}}\right)$ is left-handed (right-handed) vector coupling, and $f_{\mathrm{T}}^{\mathrm{L}}\left(f_{\mathrm{T}}^{\mathrm{R}}\right)$ tensor couplings. For SM $f_{\mathrm{V}}^{\mathrm{L}}=V_{\mathrm{tb}}, f_{\mathrm{V}}^{\mathrm{R}}=f_{\mathrm{T}}^{\mathrm{L}}=f_{\mathrm{T}}^{\mathrm{R}}=0$. In our analysis [7], we consider two scenarios: $\left(f_{\mathrm{V}}^{\mathrm{L}}, f_{\mathrm{V}}^{\mathrm{R}}\right)$ and $\left(f_{\mathrm{V}}^{\mathrm{L}}, f_{\mathrm{T}}^{\mathrm{L}}\right)$ with other couplings set to zero. The kinematics and angular distributions significantly change in the presence of anomalous Wtb couplings, both in the production and in the decay of the top quark.

We train various Bayesian Neural Networks (BNN) to discriminate between background, SM signal and anomalous couplings, and set limits based on these distributions. This analysis is performed with $7 \mathrm{TeV}$ data.

On Figure 8 we can see the 2-dimensional limits, which agree with SM prediction. Onedimensional constraints on anomalous parameters obtained by fixing one parameter to its SM value and setting the exclusion limit on the other one. For the $\left(f_{\mathrm{V}}^{\mathrm{L}}, f_{\mathrm{V}}^{\mathrm{R}}\right)$ scenario, we get $f_{\mathrm{V}}^{\mathrm{L}}>0.90(0.88)$ observed (expected) and $f_{\mathrm{V}}^{\mathrm{R}}<0.34(0.39)$, while the $\left(f_{\mathrm{V}}^{\mathrm{L}}, f_{\mathrm{T}}^{\mathrm{L}}\right)$ scenario results in the limits $f_{\mathrm{V}}^{\mathrm{L}}>$ $0.92(0.88)$ and $f_{\mathrm{T}}^{\mathrm{L}}<0.09(0.16)$.

The analysis also sets limits on FCNC anomalous couplings. FCNC tcg and tug interactions can be written in a model-independent form with the following vertex in the effective Lagrangian

$$
\frac{\kappa_{\mathrm{tqg}}}{\Lambda} g_{s} \bar{f} \sigma^{\mu \nu} \frac{\lambda^{\mathrm{a}}}{2} t G_{\mu \nu}^{\mathrm{a}}
$$



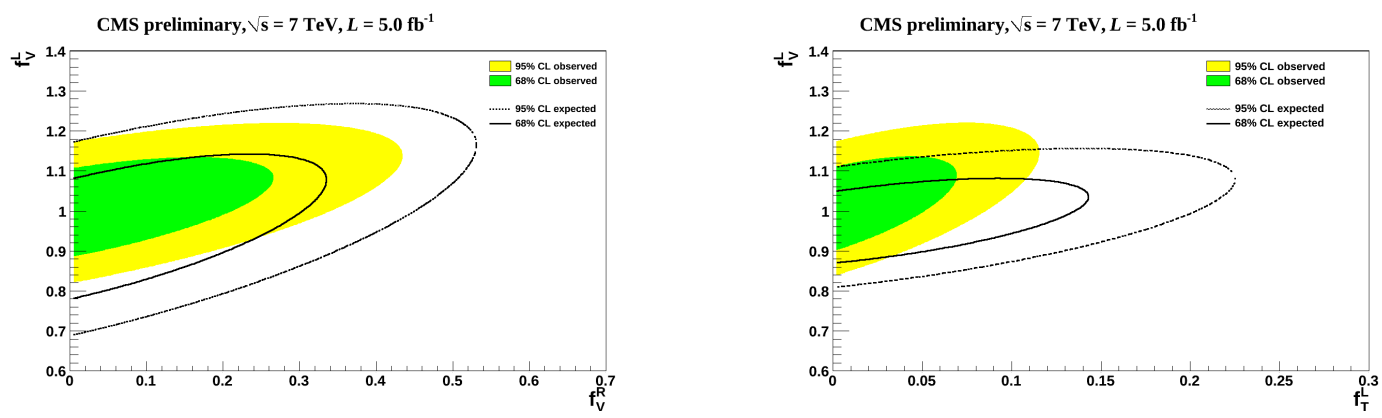

Figure 8: 2-dimensional limits for tqg couplings. $\left(f_{\mathrm{V}}^{\mathrm{L}}, f_{\mathrm{V}}^{\mathrm{R}}\right)$ scenario on the left, $\left(f_{\mathrm{V}}^{\mathrm{L}}, f_{\mathrm{T}}^{\mathrm{L}}\right)$ on the right.

where $\Lambda=\mathscr{O}(1 \mathrm{TeV})$ is the scale of new physics and $\kappa_{\mathrm{tgg}}$ defines the strength of the FCNC interactions in the tug or tcg vertices. Cross section of the single top quark production through FCNC is proportional to $\left(\kappa_{\mathrm{tgg}} / \Lambda\right)^{2}$.

The observed (expected) limits are $\frac{\kappa_{\text {tug }}}{\Lambda}<1.8 \cdot 10^{-2}\left(1.2 \cdot 10^{-2}\right) \mathrm{TeV}^{-1}$ and $\frac{\kappa_{\mathrm{cg}}}{\Lambda}<5.6 \cdot 10^{-2}(3.1$. $\left.10^{-2}\right) \mathrm{TeV}^{-1}$.

\section{Conclusion}

In conclusion, we have performed the first measurement of $\mathrm{W}$ boson helicity fractions as well as the first measurement of top quark polarization in single top production. We have improved limits for $t u(c) \gamma$ couplings and set limits on anomalous Wtb and FCNC couplings. No deviation from Standard Model has been observed. We have seen that single top at the LHC is a unique testing ground for top quark properties, complementary to tt.

\section{References}

[1] CMS Collaboration, "The CMS experiment at the CERN LHC", JINST 3 (2008) S08004.

[2] V. Khachatryan et al., "Measurement of the $\mathrm{W}$ boson helicity in events with a single reconstructed top quark in pp collisions at $\sqrt{s}=8 \mathrm{TeV}$ ", JHEP 01 (2015).

[3] J. A. Aguilar-Saavedra and J. Bernabeu, "W polarisation beyond helicity fractions in top quark decays", Nucl. Phys. B840 (2010) 349-378.

[4] CMS Collaboration, "Measurement of top quark polarization in t-channel single-top production", CMS Physics Analysis Summary CMS-PAS-TOP-13-001, CERN, Geneva, 2013.

[5] L. Lyons, D. Gibaut, and P. Clifford, "How to combine correlated estimates of a single physical quantity", Nucl. Instrum. Meth. A270 (1988) 110.

[6] CMS Collaboration, "Search for anomalous single top quark production in association with a photon", CMS Physics Analysis Summary CMS-PAS-TOP-14-003, CERN, Geneva, 2014.

[7] CMS Collaboration, "Search for anomalous Wtb couplings and top FCNC in t-channel single-top-quark events", CMS Physics Analysis Summary CMS-PAS-TOP-14-007, CERN, Geneva, 2014. 